\title{
Adult Neurogenesis: An Evolutionary Perspective
}

\author{
Gerd Kempermann \\ German Center for Neurodegenerative Diseases (DZNE) Dresden, and CRTD-Center for Regenerative \\ Therapies Dresden at Technische Universität Dresden, 01307 Dresden, Germany \\ Correspondence: gerd.kempermann@crt-dresden.de
}

When adult neurogenesis was discovered in the mammalian brain it was often considered an atavism and, even today, many people are convinced that there has been a "phylogenetic reduction" away from lifelong neurogenesis, favoring stability for complex brains. Adult neurogenesis is found throughout the animal kingdom but varies to a large extent. Mammals might have fewer neurogenic zones than, for example, fish, but within their remaining neurogenic zones, the new neurons are highly functional. Especially, humans have very substantial quantities of neurogenesis in their hippocampus. At least for the mammalian dentate gyrus, one can thus argue that there has been evolution toward neurogenesis-based plasticity rather than away from it.

$\mathrm{H}_{\mathrm{i}}^{\mathrm{s}}$ ow did adult neurogenesis, as we find it in modern mammals, including humans, evolve? What exactly is the genealogy of adult neurogenesis? Comparative studies can help guide the way in finding first answers to these questions, which are far from being merely academic. Work by Irmgard Amrein (2015) has provided an extensive update on the current information on how adult neurogenesis compares among mammalian species. Bergmann et al. (2015), Ganz and Brand (2015), and Brenowitz and Larson (2015) dealt with fish, birds, and, as a special mammalian case, humans. Arguably, the consequences of adult neurogenesis for humans are the most critical aspect because, ultimately, it is the applications to the human situation that motivates a substantial part of the research dedicated to the new neurons. There has been a long, at parts rather shallow, learning curve up to our present conviction that adult neurogenesis is neither an atavism nor one of the many oddities that populate nature films but rather a fundamentally important part of brain plasticity.

A true understanding of what new neurons are "good for" can consequently only arise from our success in placing the process of adult neurogenesis into its appropriate evolutionary context (Kaslin et al. 2008; Treves et al. 2008; Amrein and Lipp 2009; Kempermann 2012; Peretto and Bonfanti 2014). The reason for this is that the existence of adult neurogenesis still appears as somewhat counterintuitive and is in opposition to long-held scientific wisdom. Models of mind and brain did quite well without having to consider adult neurogenesis. By breaking the isolation of the new finding as particular to this or that species, the bigger picture might now help to integrate adult neurogenesis

Editors: Fred H. Gage, Gerd Kempermann, and Hongjun Song

Additional Perspectives on Neurogenesis available at www.cshperspectives.org

Copyright (C) 2016 Cold Spring Harbor Laboratory Press; all rights reserved; doi: 10.1101/cshperspect.a018986

Cite this article as Cold Spring Harb Perspect Biol 2016;8:a018986 
into more general theories on how the brain works.

To consider the bits and pieces just by themselves, in contrast, continues to lead to many paradoxical results, because we do not immediately grasp what are the rules and what are the exceptions. Perhaps there will never be one unifying theory, but that, again, only a comprehensive consideration across the full spectrum of phenomena related to adult neurogenesis will tell.

Adult neurogenesis in mammals is a complex process, requiring intricate control and regulation, is energetically expensive, and also bears certain risk. For example, stem and progenitor cells seem to be a potential source of gliomas (and possibly the rare neuronal tumors of the brain), aberrant wiring of newborn neurons might contribute to epilepsy, and more subtle errors of development might be disadvantageous in numerous cognitive functions. The price of this risk is presumably paid for something. Adult neurogenesis is already effective early in life, actually very well before true adulthood, and is at very high levels when sexual maturity has been reached. Behavioral advantages associated with adult neurogenesis must be relevant during the reproductive period because then the trait will underlie direct selective pressure. With such thoughts, one already goes one step further by trying to deduce a mechanism from the comparative analysis. This is inspiring and good "food for thought" but remains quite speculative at this time.

Therefore, what is here called an "evolutionary perspective" can really be only a first step and quite literally only a perspective in the sense of a directional view. Developing the idea that there has been an evolutionary process leading to adult hippocampal neurogenesis, as we find it in contemporary mammals, requires a large interdisciplinary effort that includes the involvement of evolutionary and theoretical biologists along developmental and behavioral neuroscientists as well as possibly other disciplines. Freehand evolutionary considerations tend to be overly simplistic and run the danger of leading to untrue face value validity. On the other hand, without the first steps, the more advanced ones are unlikely to be made. The central hypothesis is quite straightforward. Mammalian adult hippocampal neurogenesis is a trait shaped by evolutionary forces that have contributed to the advantages in natural selection that are associated with the mammalian dentate gyrus. To confirm (or refute) this hypothesis, however, extensive comparative studies will be instrumental. "Evolutionary" here means foremost to start looking across species boundaries and begin to ask whether the differences that are observed do tell us about functional relevance both in terms of ontogeny and phylogeny. Although the hypothesis has been formulated in a traditional way, it should be noted that there are important arguments for questioning the sufficiency of natural selection alone to mechanistically explain how traits evolved. Be that as it may, even the description of adult neurogenesis across the animal kingdom alone will help us to understand better how adult neurogenesis relates to functionality and the cognitive demands arising from the living conditions of different species.

Intriguingly, at least to my knowledge, the very first scientific meeting that was solely dedicated to adult neurogenesis focused on comparative studies. Martin Wojtowicz from Toronto, who had been invited to that conference, identified the central issue of how the findings on mammalian adult neurogenesis, which to that date had been obtained only in laboratory animals, related to feral conditions (Boonstra et al. 2001; Barker et al. 2005). He rightfully felt that the significance of the emerging field of neurogenesis would critically depend on the demonstration that it has relevance in "real life."

At that time, however, very little was known about "adult neurogenesis in wild populations" and, although the situation has much improved (cf. Amrein 2015), the many difficulties in studying adult neurogenesis in the wild have prevented getting anywhere near the depth and scope of the studies that are possible in research animals.

Nevertheless, today we can be sure beyond reasonable doubt that adult neurogenesis is not a laboratory artifact (Patzke et al. 2013). The 
question, however, remains open whether and how living in cages for hundreds of generations has changed adult neurogenesis, overemphasizing some aspects, whereas obscuring others. Comparative studies and evolutionary considerations, as difficult as they are, allow putting study results into a larger perspective. In laboratory animals, there might have been some evolution in its own right (at least genetic drifts are well described) or some regression to a less advanced state, but we know that the principles are maintained.

That adult neurogenesis is an atavism with no or little relevance for today's species and, thus, as it is sometimes said, something like "the appendix of the brain," has been many people's tacit, or sometimes explicit, assumption for many years. Since Pasko Rakic's influential paper of 1985, "Limits of Neurogenesis in Adult Primates," (Rakic 1985) in which he had argued that advanced brains would favor stability over plasticity and that new neurons would disrupt complex networks, there must have been a certain fear that demonstrating adult neurogenesis in humans would put us at the same level with lobsters, rodents, and birds. Today, adult hippocampal neurogenesis in humans has been proven beyond doubt and the question has been turned around (Eriksson et al. 1998; Knoth et al. 2010; Spalding et al. 2013). Why is adult neurogenesis there? How did it evolve? What is similar to and what is different from other species. Related is the question of how different adult hippocampal neurogenesis is from neurogenesis in other brain regions, which is found in most species but not in humans. To be fair, Rakic's argument primarily aimed at cortical neurogenesis but the hippocampus is an archicortical structure and was not explicitly excluded from the argument.

There is the somewhat vague concept of the "phylogenetic reduction" that supposedly governs adult neurogenesis on a phylogenic scale; although the evidence in favor of the idea that neurogenesis is lost in the course of evolution has been at best anecdotal (Kaslin et al. 2008). What is true is that the brains of many lower vertebrates have a much greater potential for regeneration than mammals with, for example, zebrafish (and presumably other gymnotiform fish) being true masters of restoration after damage (Grandel et al. 2006; Kaslin et al. 2008). What has not been particularly clear is the link that might exist between regenerative neurogenesis and the type of adult neurogenesis we find in mammals today and which does not or hardly contribute to regeneration but is profoundly involved in plasticity. Consequently, if there is something like a phylogenetic reduction, it cannot apply to adult hippocampal neurogenesis. Nonmammalian vertebrates do not have a dentate gyrus of the same structure as mammals have (Treves et al. 2008) and, as far as the few available data, are conclusive; "hippocampal" neurogenesis in these species does not seem to differ from neurogenesis elsewhere in the brain (but see discussion in Barnea and Nottebohm 1994). Adult hippocampal neurogenesis in mammals originates from an ectopic precursor cell population that resides in a defined stem-cell niche detached from the ventricular wall. No other example of such an ectopic niche is known. At least to our present knowledge, all other known neurogenic niches are related to the ventricular wall.

Even the two canonical neurogenic regions of the adult mammalian brain differ by many more features than they share. Neurogenesis in the adult olfactory bulb generates a diverse range of interneurons, and is involved in the processing of sensory input (see Lim and Alvarez-Buylla 2015). In contrast, adult hippocampal neurogenesis produces only one type of excitatory principal neuron and plays a role in flexible memory formation. The two types of adult neurogenesis originate from differently regionalized precursor cell populations: a "dorsal" pattern in the hippocampus and a "ventral" regionalization in the subventricular zone (SVZ)/olfactory bulb system. These few exemplary differences alone might suggest that the two forms of mammalian adult neurogenesis evolved to cope with substantially different challenges and demands, and were shaped by quite different evolutionary forces. The neurogenesis in the olfactory system (i.e., the system of chemical senses) is evolutionarily old and highly conserved, whereas the dentate gyrus, 
in which adult hippocampal neurogenesis occurs, is a "young" substructure (in an "old" archicortical brain region) and in its connectivity and specialized function is unique to mammals, even though related structures are, for example, found in fish (Ganz et al. 2014). Intriguingly, it turns out that this "late" adult neurogenesis is preserved in the central nervous system of humans. It has been argued for some time that adult neurogenesis must be an atavism or, at best, a heritage from our ancestors (and this might indeed be true for the olfactory system), but it turns out that this explanation does not fit for the hippocampus.

In general terms, plasticity of the central nervous system (i.e., the reciprocal influences of structure and function) is a means of adaptability. A qualifying feature of advanced brains is their plasticity, which is how they can adjust their network structure to actual conditions. Many primitive nervous systems are hardwired. It is still conceivable that, in such situations, single neurons can be replaced by adult neurogenesis, but the circumstances are considerably different from the neurogenesis related to plasticity. One might say that primitive adult neurogenesis is a way of maintaining stability, whereas advanced adult neurogenesis is a means for change.

Gerald Edelman and others have likened the processes of plasticity underlying essentially all functions that we can remotely call "learning" to the mechanisms that supposedly govern evolution itself and coined the term "neural Darwinism" (Edelman 1993). The term might not be entirely fitting because it is not clear to which degree it is a selection from random connections that underlie the formation of adaptive circuitry, and the idea has also been criticized for other reasons. But plasticity is indeed a selection process. At the synaptic level, connections that are used are strengthened, whereas those that are not used are abandoned. Adult neurogenesis adds another layer of plasticity in that new nodes are recruited to the network. And in adult hippocampal neurogenesis, too, a surplus of new neurons is generated first from which only a subset survives. And it is exactly these new neuronal nodes that, at least for a transient period, are the carriers of synaptic plasticity (Snyder et al. 2001; SchmidtHieber et al. 2004; Garthe et al. 2009). For a number of weeks after they were born, the new neurons have a lower threshold for longterm potentiation. This directs the action to the new cells and results in a bias toward the most plastic cells in the local circuitry.

It is not clear how exactly all of this relates to the situation in the olfactory bulb, in which information on the network physiology is scarcer. The relationship to olfactory learning appears to be much more indirect as, here, neurogenesis is found at the first and second relay stations of sensory afferents to the brain but not beyond, for example, in the piriform cortex (for details, see Lledo and Valley 2015). In mammals, it is again found in the hippocampus, which also integrates olfactory information into weaving the contexts of the information processed there. Consequently, in those mammalian species that command both sites of adult neurogenesis, there is a partial functional link between the two kinds of neurogenesis. In adult humans, neither adult olfactory bulb neurogenesis nor, therefore, this functional link appears to be preserved. In any case, from what we know today, it seems that olfactory bulb neurogenesis is primarily involved in maintaining network function in the face of ever-changing input organization rather than actively providing adaptation. Nevertheless, there is a component of structural and functional plasticity here as well and in both neurogenic zones; adult neurogenesis is more related to physiological processes than to regeneration or restoration after damage.

Besides the often impressive capability for regenerative neurogenesis, we know only little about the contribution of adult neurogenesis to baseline plasticity in lower vertebrates. One example is the increase in mushroom body neurogenesis in crickets after exposure to an enriched environment. Similarly, olfactory neurogenesis in crustaceans was stimulated by environmental enrichment. It is not clear what this kind of plasticity means in terms of functional consequences, but it seems that adult neurogenesis provides a means of structural adaptability 
in many species, not only mammals. Birds provide one of the most impressive examples. Continuous neurogenesis can be found throughout the songbird brain, and part of it could be directly linked to plasticity. Adult male canaries produce neurons in a seasonal pattern as they learn and forget their songs. For years, this has been the most visible and intriguing demonstration that new neurons are directly tied into brain function. There has also been some indication that neurogenesis in the bird equivalent of the hippocampus can be related to memory formation in food-caching birds, but the data are a bit less clear, partly because we know comparatively little about the bird hippocampus.

Nevertheless, what we witness in the bird or fish "hippocampus" might be similar to the predecessors of adult neurogenesis in the mammalian hippocampus (Barnea and Nottebohm 1994; Ganz et al. 2014). If we look at adult neurogenesis of species living today, we obviously can only infer what the neurogenic potential of common ancestors might have been. But judging from the appearance of the hippocampus and its equivalents in today's species, the mammalian hippocampus is clearly set apart by the existence of a dentate gyrus of particular connectivity and presumably function (Treves et al. 2008; Kempermann 2012). This rather strong statement is based on a still rather superficial data set, because there are too few direct comparative studies, especially regarding the exact connectivity. Here, future research needs to set in. We are in urgent need of more detailed descriptions of adult neurogenesis in many more species than we have available today. Such data sets could easily be related to what else is known about those species, their living conditions, their behaviors, and the like (Galsworthy et al. 2005). Adult neurogenesis thereby could be placed into an ethological context (Amrein and Lipp 2009). This would not immediately explain how adult neurogenesis evolved but it would allow clearer insight into what we should mean if we say that adult hippocampal neurogenesis is "regulated by environment and behavior."

But if adult hippocampal neurogenesis indeed contributes to the individual's adapt- ability in response to the challenges of change, novelty, and complexity (in my view, specifically through the flexible integration of novel information into preexisting representations [Garthe et al. 2009]), the same (or at least something very similar) might apply to species as a whole. We would predict that highly adaptive species with larger and more variable territories rely more on adult hippocampal neurogenesis than species with more limited and stable habitats (Kempermann 2012). When this idea was first published, some comments criticized it by taking it to extremes. But we obviously cannot say that we became what we are only because of some new neurons in our brain, and one should refrain from all extrapolations linking adult neurogenesis directly to specific behaviors or even historic events. But that truth applies to all evolutionary perspectives; their metaphorical power can be large and deceiving. Description and explanation are not to be confused.

This is emphasized here because the trait "adult neurogenesis" poses some serious challenges to at least simple evolutionary explanations based on natural selection. It is a highly polygenic trait, and numerous genes have already been identified to ultimately have essentially identical effects on net neurogenesis (Overall et al. 2012). This is evident from the literature on knockout mice, many of which have surprisingly stereotypic phenotypes, but even more so from studies on between-strain polymorphisms in genetic reference populations (Kempermann et al. 2006). Recombinant inbred lines of mice are essentially inbred strains derived from brother-sister matings at the F2 level of an initial cross between two parental strains. Across the strains, the shuffling of the parental polymorphisms results in an almost normal distribution of phenotypes with respect to neurogenesis. Selection, however, would select "for phenotypes," so the question is how genetic variation in single genes (or many genes) would be able to exert a phenotypic change in neurogenesis that can provide a large enough advantage to be selected for. As McKinney and Gittleman (1995) stated: "Ontogenies evolve, not genes or adults." This might be true, except 


\section{G. Kempermann}

for the case that ontogeny stretches out into adulthood and development "never ends" as it happens in adult neurogenesis.

Adult neurogenesis is also an individualizing trait (Freund et al. 2013). Even before an identical genetic background, subtle individual differences in starting conditions and differential behavioral trajectories result in an increase in phenotypic variation with time, also during the reproductive period. Such caveats are not unique to adult neurogenesis, but stress that evolution is more than meets the eye in a first "perspective," no matter how important and informative that will be. In summary, evolutionary considerations on adult neurogenesis do not stop with comparative studies or speculations about its "why" and "how." There are fundamental issues to be resolved for a quantitative trait that is like no other.

\section{REFERENCES}

${ }^{*}$ Reference is also in this collection.

* Amrein I. 2015. Adult hippocampal neurogenesis in natural populations of mammals. Cold Spring Harb Perspect Biol 7: a021295.

Amrein I, Lipp HP. 2009. Adult hippocampal neurogenesis of mammals: Evolution and life history. Biol Lett 5: 141144.

Barker JM, Wojtowicz JM, Boonstra R. 2005. Where's my dinner? Adult neurogenesis in free-living food-storing rodents. Genes Brain Behav 4: 89-98.

Barnea A, Nottebohm F. 1994. Seasonal recruitment of hippocampal neurons in adult free-ranging blackcapped chickadees. Proc Natl Acad Sci 91: $11217-$ 11221.

* Bergmann O, Spalding KL, Frisén J. 2015. Adult neurogenesis in humans. Cold Spring Harb Perspect Biol 7: a018994.

Boonstra R, Galea L, Matthews S, Wojtowicz JM. 2001. Adult neurogenesis in natural populations. Can J Physiol Pharmacol 79: 297-302.

* Brenowitz EA, Larson TA. 2015. Neurogenesis in the adult avian song control system. Cold Spring Harb Perspect Biol 7: a019000.

Edelman GM. 1993. Neural Darwinism: Selection and reentrant signaling in higher brain function. Neuron 10: $115-125$.

Eriksson PS, Perfilieva E, Björk-Eriksson T, Alborn AM, Nordborg C, Peterson DA, Gage FH. 1998. Neurogenesis in the adult human hippocampus. Nat Med 4: 13131317.
Freund J, Brandmaier AM, Lewejohann L, Kirste I, Kritzler M, Kruger A, Krüger A, Sachser N, Lindenberger U, Kempermann G. 2013. Emergence of individuality in genetically identical mice. Science 340: 756-759.

Galsworthy MJ, Amrein I, Kuptsov PA, Poletaeva II, Zinn P, Rau A, Vyssotski A, Lipp HP. 2005. A comparison of wild-caught wood mice and bank voles in the IntelliCage: Assessing exploration, daily activity patterns and place learning paradigms. Behav Brain Res 157: 211 217.

* Ganz J, Brand M. 2015. Adult neurogenesis in fish. Cold Spring Harb Perspect Biol doi: 10.1101/cshperspect .a019018.

Ganz J, Kroehne V, Freudenreich D, Machate A, Geffarth M, Braasch I, Kaslin J, Brand M. 2014. Subdivisions of the adult zebrafish pallium based on molecular marker analysis. F1000Res 3: 308.

Garthe A, Behr J, Kempermann G. 2009. Adult-generated hippocampal neurons allow the flexible use of spatially precise learning strategies. PLoS ONE 4: e5464.

Grandel H, Kaslin J, Ganz J, Wenzel I, Brand M. 2006. Neural stem cells and neurogenesis in the adult zebrafish brain: Origin, proliferation dynamics, migration and cell fate. Dev Biol 295: 263-277.

Kaslin J, Ganz J, Brand M. 2008. Proliferation, neurogenesis and regeneration in the non-mammalian vertebrate brain. Philos Trans $R$ Soc Lond B Biol Sci 363: $101-122$.

Kempermann G. 2012. New neurons for "survival of the fittest." Nat Rev Neurosci 13: 727-736.

Kempermann G, Chesler EJ, Lu L, Williams RW, Gage FH. 2006. Natural variation and genetic covariance in adult hippocampal neurogenesis. Proc Natl Acad Sci 103: 780785.

Knoth R, Singec I, Ditter M, Pantazis G, Capetian P, Meyer RP, Horvat V, Volk B, Kempermann G. 2010. Murine features of neurogenesis in the human hippocampus across the lifespan from 0 to 100 years. PLoS ONE 5: e8809.

* Lim DA, Alvarez-Buylla A. 2015. The adult ventricularsubventricular zone (V-SVZ) and olfactory bulb neurogenesis. Cold Spring Harb Perspect Biol doi: 10.1101/ cshperspect.a018820.

* Lledo P-M, Valley M. 2015. Adult olfactory bulb neurogenesis. Cold Spring Harb Perspect Biol doi: 10.1101/cshper spect.a018945.

McKinney ML, Gittleman JL. 1995. Ontogeny and phyologeny: Tinkering with covariation in life history, morphology and behaviour. In Evolutionary change and heterochrony (ed. McNamara K), pp. 21-47. Wiley, New York.

Overall RW, Paszkowski-Rogacz M, Kempermann G. 2012. The mammalian adult neurogenesis gene ontology (MANGO) provides a structural framework for published information on genes regulating adult hippocampal neurogenesis. PLoS ONE 7: e48527.

Patzke N, Spocter MA, Karlsson KÆ, Bertelsen MF, Haagensen M, Chawana R, Streicher S, Kaswera C, Gilissen E, Alagaili AN, et al. 2013. In contrast to many other mammals, cetaceans have relatively small hippocampi that 
Evolution of Adult Neurogenesis

appear to lack adult neurogenesis. Brain Struct Funct 220: 361-383.

Peretto P, Bonfanti L. 2014. Major unsolved points in adult neurogenesis: Doors open on a translational future? Front Neurosci 8: 154.

Rakic P. 1985. Limits of neurogenesis in primates. Science 227: 1054-1056.

Schmidt-Hieber C, Jonas P, Bischofberger J. 2004. Enhanced synaptic plasticity in newly generated granule cells of the adult hippocampus. Nature 429: 184-187.
Snyder JS, Kee N, Wojtowicz JM. 2001. Effects of adult neurogenesis on synaptic plasticity in the rat dentate gyrus. $J$ Neurophysiol 85: 2423-2431.

Spalding KL, Bergmann O, Alkass K, Bernard S, Salehpour M, Huttner HB, Boström E, Westerlund I, Vial C, Buchholz BA, et al. 2013. Dynamics of hippocampal neurogenesis in adult humans. Cell 153: 1227.

Treves A, Tashiro A, Witter ME, Moser EI. 2008. What is the mammalian dentate gyrus good for? Neuroscience 154: $1155-1172$. 


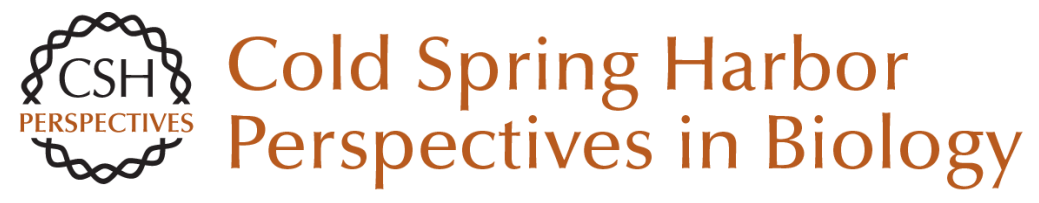

\section{Adult Neurogenesis: An Evolutionary Perspective}

Gerd Kempermann

Cold Spring Harb Perspect Biol 2016; doi: 10.1101/cshperspect.a018986 originally published online December 18, 2015

\section{Subject Collection Neurogenesis}

Adult Neurogenesis and Psychiatric Disorders Eunchai Kang, Zhexing Wen, Hongjun Song, et al.

Neuronal Circuitry Mechanisms Regulating Adult Mammalian Neurogenesis Juan Song, Reid H.J. Olsen, Jiaqi Sun, et al.

Neurogenesis in the Developing and Adult Brain

--Similarities and Key Differences

Magdalena Götz, Masato Nakafuku and David Petrik

Genetics and Epigenetics in Adult Neurogenesis Jenny Hsieh and Xinyu Zhao

The Adult Ventricular-Subventricular Zone (V-SVZ) and Olfactory Bulb (OB) Neurogenesis Daniel A. Lim and Arturo Alvarez-Buylla

Diversity of Neural Precursors in the Adult Mammalian Brain Michael A. Bonaguidi, Ryan P. Stadel, Daniel A. Berg, et al.

Detection and Phenotypic Characterization of Adult Neurogenesis $H$. Georg Kuhn, Amelia J. Eisch, Kirsty Spalding, et al.

Maturation and Functional Integration of New Granule Cells into the Adult Hippocampus Nicolas Toni and Alejandro F. Schinder
Adult Olfactory Bulb Neurogenesis

Pierre-Marie Lledo and Matt Valley

Adult Neurogenesis in Fish Julia Ganz and Michael Brand

In Vitro Models for Neurogenesis Hassan Azari and Brent A. Reynolds

Engineering of Adult Neurogenesis and Gliogenesis

Benedikt Berninger and Sebastian Jessberger

Computational Modeling of Adult Neurogenesis James B. Aimone

Control of Adult Neurogenesis by Short-Range Morphogenic-Signaling Molecules Youngshik Choe, Samuel J. Pleasure and Helena Mira

Adult Neurogenesis: An Evolutionary Perspective Gerd Kempermann

Epilepsy and Adult Neurogenesis

Sebastian Jessberger and Jack M. Parent

For additional articles in this collection, see http://cshperspectives.cshlp.org/cgi/collection/

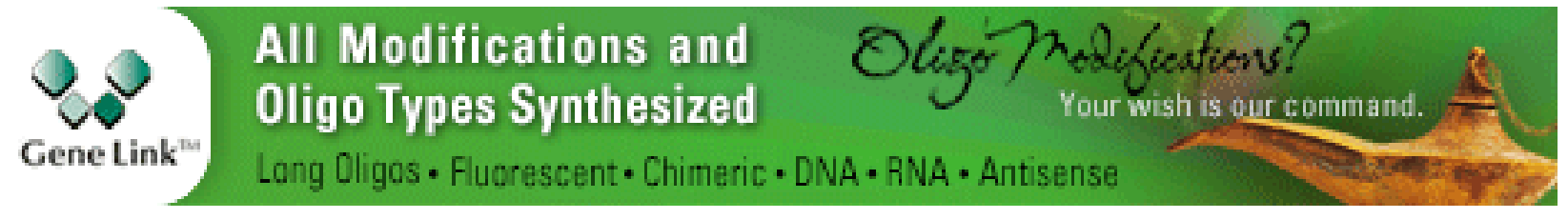


For additional articles in this collection, see http://cshperspectives.cshlp.org/cgi/collection/

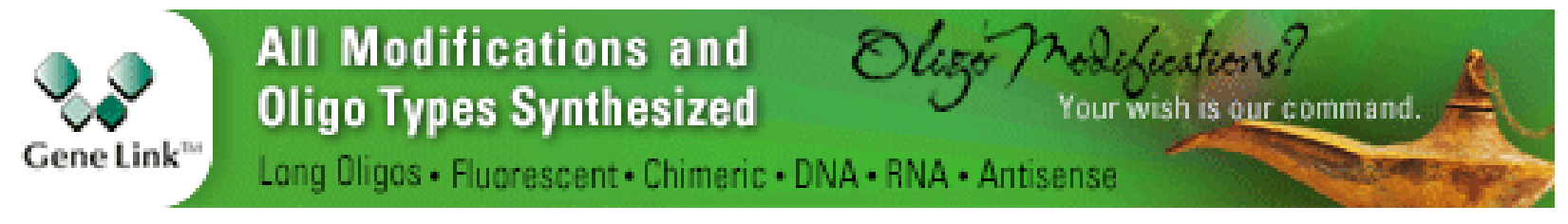

Copyright @ 2016 Cold Spring Harbor Laboratory Press; all rights reserved 